\title{
Rectangle of Influence Drawings of Graphs without Filled 3-Cycles ${ }^{\star}$
}

\author{
Therese Biedl ${ }^{1 \star \star}$, Anna Bretscher ${ }^{2}$, and Henk Meijer ${ }^{2}$ \\ 1 Department of Computer Science University of Waterloo \\ Waterloo, Ontario N2L 3G1, Canada \\ biedl@uwaterloo.ca \\ 2 Computing and Information Science Department Queen's University \\ Kingston, Ontario K7L 3N6, Canada \\ \{bretscha, henk\}@qucis .queensu.ca
}

\begin{abstract}
In this paper, we study rectangle of influence drawings, i.e., drawings of graphs such that for any edge the axis-parallel rectangle defined by the two endpoints of the edge is empty. Specifically, we show that if $G$ is a planar graph without filled 3-cycles, i.e., a planar graph that can be drawn such that the interior of every 3-cycle is empty, then $G$ has a rectangle of influence drawing.
\end{abstract}

\section{Introduction}

Let $G=(V, E)$ be a planar graph. A planar straight-line drawing of $G$ is a mapping of the vertices to points in the two-dimensional grid such that no two edges intersect (except at common endpoints) and no edge intersects a vertex other than its endpoints. It has been known for a long time that every planar graph has a planar straight-line drawing; see e.g. [6]. In this paper, we are interested in straight-line drawings that are also proximity drawings: an edge in the drawing implies that the two endpoints of the edge are "close" by some definition. In this paper, we study a type of proximity drawings known as rectangle of influence drawings, where an edge implies that the axis-parallel rectangle defined by its endpoints contains no other points of the drawing.

Existing results A recent survey of proximity drawings can be found in [5]. The notion of rectangular visibility where two points "see" each other if and only if their rectangle of influence is empty, was first defined in [7]. Alon et al. call two points box separated if and only if their closed rectangle of influence is empty. They constructed an upper bound on the graph of all box separated points, i.e. on the closed rectangle of influence graph [1]. Rectangle of influence graphs were then investigated in [9] with respect to the data analysis problem and later extended by [4] such that two sets of points are given, $P$ and $O$, in

\footnotetext{
* The results are part of the second author's Master's thesis at Queen's University.

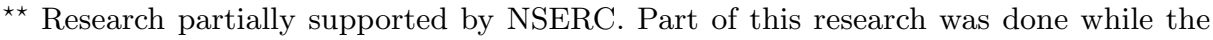
author was at McGill University.
} 
which $p_{1}, p_{2} \in P$ see each other if and only if their rectangle of influence contains no points from $O$.

In [8], Liotta et al. examined rectangle of influence drawings with respect to graph characterization or the proximity drawing problem. In particular, they investigate whether several subclasses of planar graphs as well as complete graphs admit rectangle of influence drawings. They study both the model where the rectangle of influence is closed (which is what we mainly consider in this paper), and also the model where the rectangle of influence is open.

In all existing works, only strong rectangle of influence drawings have been studied; i.e., there is an edge $(v, w)$ in the drawing if and only if the axis-parallel rectangle defined by the points assigned to $v$ and $w$ is empty except for these two points. As opposed to this, we will allow weak rectangle of influence drawings, where edges are optional, i.e., need not be added if the rectangle of influence is empty.

Our results We study weak planar rectangle of influence drawings and show that a large class of planar graphs has such a drawing. More precisely, if $G$ is a planar graph that has a planar drawing such that the interior of any 3-cycle is empty (we call this a drawing of $G$ without filled 3-cycle), then $G$ has a weak planar rectangle of influence drawing. Since in any planar closed rectangle of influence drawing, any 3-cycle must be empty, the converse also holds: if $G$ is a planar graph, then it has a weak planar closed rectangle of influence drawing if and only if it can be drawn without filled 3-cycle.

Note that a drawing without filled 3-cycle is the same as a drawing where the outer-face is not a 3-cycle and that has no separating 3-cycle (that is, a 3-cycle such that both inside and outside the cycle are other vertices). Testing whether a graph can be embedded without a separating 3-cycle can be done in $O(n)$ time [2], and a slight modification of this algorithm yields an $O(n)$ time test whether a planar graph has a drawing without filled 3-cycles.

\section{Definitions}

We assume familiarity with basic graph theoretic and geometric terminology (see also [3] and [10]).

A graph is called planar if it can be drawn in the plane without an edge crossing. A specific planar drawing splits the plane into components called faces; the unbounded face is called the outer-face. A planar graph is called triangulated if all faces are 3-cycles, and internally triangulated if all faces except the outerface are 3-cycles.

If a planar drawing is fixed, then we call a 3-cycle filled if there is a vertex inside the drawing of the 3 -cycle. If $G$ is a planar graph that has a planar drawing such that there are no filled 3-cycles, then we call $G$ an NF3-graph.

A straight-line embedding of a graph $G=(V, E)$ is an injective mapping $p: V \rightarrow R^{2}$ such that for any edge $(v, w)$ and any vertex $x$, the open line segment $(p(v), p(w))$ does not intersect $p(x)$. It is called a planar straight-line embedding if additionally for any pair of edges $(v, w),\left(v^{\prime}, w^{\prime}\right)$ the open line segments $(p(v), p(w))$ and $\left(p\left(v^{\prime}\right), p\left(w^{\prime}\right)\right)$ do not intersect. For ease of notation, we 
will drop the use of the function $p$, and use graph-theoretic terms such as vertex to denote both the graph-theoretic object and its representation in a drawing.

Given two distinct points $p, p^{\prime}$ in the plane, $R\left[p, p^{\prime}\right]$ denotes the minimal axisaligned rectangle containing both $p$ and $p^{\prime}$. We call $R\left[p, p^{\prime}\right]$ the closed rectangle of influence of $p, p^{\prime}$. If $R\left[p, p^{\prime}\right]$ is a non-degenerate rectangle, then we denote by $R\left(p, p^{\prime}\right)$ the interior of $R\left[p, p^{\prime}\right]$. If $R\left[p, p^{\prime}\right]$ is a line segment, then we denote by $R\left(p, p^{\prime}\right)$ the open line segment between $p$ and $p^{\prime}$. We call $R\left(p, p^{\prime}\right)$ the open rectangle of influence of $p, p^{\prime}$. Let $P$ be a set of points. If we take $P$, together with all line segments $\left[p, p^{\prime}\right]$ (where $p, p^{\prime} \in P$ ) for which the open/closed rectangle of influence contains no points of $P$ other than $p$ and $p^{\prime}$, then we obtain a straightline drawing of some graph, called the strong open/closed rectangle of influence graph of $P$. We say that a graph $G$ has a weak open/closed rectangle of influence drawing if there exists a one-to-one mapping $V \rightarrow P \subset R^{2}$ such that $G$ is a subgraph of the strong open/closed rectangle of influence graph of $P$. As we will not study strong rectangle of influence drawings further, for easier notation we will drop "weak" for the remainder of this paper. We also use the term oRIdrawing for an open rectangle of influence drawing, and $c R I$-drawing for a closed rectangle of influence drawing.

\section{Rectangle of Influence Drawings for NF3-Graphs}

Our study of NF3-graphs was motivated by the following observation:

Lemma 1. If a graph admits a planar closed rectangle of influence drawing, then it is an NF3-graph.

Proof. Assume that a graph $G$ has a planar cRI-drawing, and let $\{a, b, c\}$ be the points of a 3 -cycle of $G$. The minimum enclosing rectangle of points $\{a, b, c\}$ must be empty (except for $a, b, c$ ), because it is the union of the rectangles of influence $R[a, b], R[b, c], R[c, a]$, which must be empty because these are edges. Since the minimum enclosing rectangle also contains the straight-line drawing of the 3-cycle $\{a, b, c\}$, and since the drawing is planar, that means that no vertex can be inside the 3 -cycle in $G$. So every 3 -cycle in $G$ is empty, which means that $G$ is an NF3-graph.

In this section, we show the reverse of this lemma, i.e., we show that every NF3-graph admits a planar closed rectangle of influence drawing. We prove this first for a special class of NF3-graphs; namely, those that are biconnected and have an embedding that is internally triangulated and has no chord, i.e., no edge between two non-consecutive vertices on the outer-face. Then we show that any NF3-graph can be converted into such an NF3-graph by adding edges; this shows that any NF3-graph has a weak planar rectangle of influence drawing.

\subsection{Biconnected, Chordless, Internally Triangulated NF3-graphs}

Assume for the remainder of this section that $G$ is a biconnected NF3-graph and that an embedding of $G$ exists that has no filled 3-cycles, no chords, and is 
internally triangulated. This embedding will be used as the input for a drawing algorithm presented in this section and whenever we talk about the graph $G$, we in fact refer to this embedding. Throughout this section, we assume that $G$ has at least 4 vertices; any graph with less than 4 vertices has a planar cRIdrawing. This implies that the outer-face has at least 4 vertices, because if it had 3 vertices, it would be a filled 3 -cycle since $n \geq 4$.

Our algorithm to create a planar cRI-drawing of $G$ is similar in spirit to previous algorithms for planar straight-line drawings (e.g. [6]): add vertices to the drawing one-by-one or in small groups, and maintain an invariant that allows one to add these vertices easily. However, both the manner of selecting the next group of vertices and the invariant is different in our algorithm.

The Invariant Our invariant is, loosely speaking, that the relevant part of the outer-face of the current subgraph is drawn on a diagonal. To make this more precise, we need more definitions. To be able to state these definitions precisely, we modify the input graph.

Definition 1. Let $G_{s}$ denote a connected induced subgraph of $G=(V, E)$ and let $V_{s}$ be the set of vertices of $G_{s}$. Let $v_{0}, v_{1}, v_{2}, \ldots, v_{m-1}$ be the list of vertices on the outer-face of $G_{s}$ in order. $G_{s}$ may not be biconnected, so some vertices may appear more than once in this list. We require that any vertex in $V-V_{s}$ lies in the outer-face of $G_{s}$. An edge $(v, w)$ on the outer-face of $G_{s}$ is called an active edge if one of its incident faces is not the outer-face of $G$ and contains a vertex from $V-V_{s}$. A vertex $v_{i}$ on the outer-face of $G_{s}$ is called an internal vertex if both $\left(v_{i-1}, v_{i}\right)$ and $\left(v_{i}, v_{i+1}\right)$ are active edges and there is path from $v_{i-1}$ to $v_{i+1}$ that uses only vertices in $V-V_{s}$ (where the arithmetic in the indices is done modulo $m$ ). A ridge $R=\left\{r_{1}, r_{2}, \ldots, r_{l}\right\}$ of $G_{s}$ is a set of consecutive vertices on the outer-face of $G_{s}$ such that $R$ contains all active edges and internal vertices of $G_{s}$.

Definition 2. A set of monotonically decreasing points $i s$ a set of points $P=$ $\left\{p_{1}, p_{2}, \ldots, p_{l}\right\}$ such that for $1 \leq i<l$ we have $x\left(p_{i}\right)<x\left(p_{i+1}\right)$ and $y\left(p_{i}\right)>$ $y\left(p_{i+1}\right)$.

Definition 3. Given a subgraph $G_{s} \subset G$, a planar $c R I$-drawing $\Gamma$ of $G_{s}$ is said to satisfy the invariant if there exists a ridge $R=\left\{r_{1}, \ldots, r_{l}\right\}$ such that

- $R$ is a simple path,

- $R$ is drawn on a set of monotonically decreasing points, and

- no point in $\Gamma$ is above the ridge, i.e., if we draw the polygonal chain through points in $R$, then no point in $\Gamma$ is strictly above or to the right of a point of this polygonal chain.

For an illustration of the above definitions, see Figure 1.

Remark 1. If $\Gamma$ satisfies the invariant, then we may assume without loss of generality that the ridge is drawn on a diagonal line. 

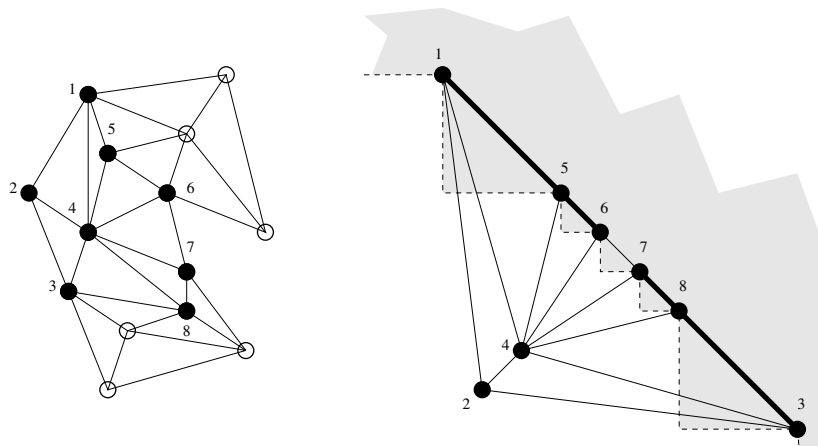

Fig. 1. Illustration of the invariant. On the left is a graph $G$ with vertices in $V_{s}$ in black; on the right is a drawing of $G_{s}$ that satisfies the invariant; the ridge $R$ consists of vertices $1,5,6,7,8$ and 3 ; the region that is known not to contain any other point of the drawing of $G_{s}$ is shaded; active edges are shown thicker and vertices 5 and 8 are internal.

Proof. Because the ridge is on a set of monotonically decreasing points, we have $x\left(r_{1}\right)<x\left(r_{2}\right)<\cdots<x\left(r_{l}\right)$ and $y\left(r_{1}\right)>y\left(r_{2}\right)>\cdots>y\left(r_{l}\right)$. It is known [8] that if we apply an order-preserving transformation to a cRI-drawing, i.e., a transformation so that the orders along the $x$-axis and along the $y$-axis are preserved, then the resulting drawing is again a cRI-drawing. One can also verify that if the initial drawing was planar, then so is the resulting drawing. Thus, we can define a transformation of $\Gamma$ that maps $r_{i}$ to $(i, l+1-i)$ and that is order-preserving. The resulting drawing then also satisfies the invariant and the ridge is drawn on a diagonal line.

Adding Vertices In this section we show how to build a drawing while maintaining the invariant. We start by initialising $V_{s}$ as a set of consecutive vertices on the outer-face of $G$. If the outer-face of $G$ has size $t$, we require that $3 \leq\left|V_{s}\right|<t$. $G_{s}$ is the subgraph induced by $V_{s}$ and the ridge $R$ contains all vertices in $V_{s}$. Since $G$ is biconnected, $R$ is a simple path. We place $r_{1}, \ldots, r_{l}$ on the diagonal $y=-x$. Since $l$ is less than the cardinality of the outer-face and $G$ has no chords, there are no edges between these vertices other than between consecutive ones, so we obtain an cRI-drawing of $G_{s}$. Notice that all edges in $G_{s}$ are active and that all vertices except $r_{1}$ and $r_{l}$ are internal. It is easy to verify that the invariant holds.

So assume now that we have a drawing $\Gamma$ of a subgraph $G_{s}$ that satisfies the invariant, and $R=\left\{r_{1}, \ldots, r_{l}\right\}$ is the ridge of this drawing. Now we show how to add more vertices to this drawing. Specifically, we will give two methods of adding vertices that clearly maintain the invariant. In the next section we then show that one of these possibilities can always be applied.

We first develop a generic placement of a point such that the point sees (that is, has an empty rectangle of influence to) many points on the ridge. For a vertex 
$v$, denote by $x(v)$ and $y(v)$ the $x$ - and $y$-coordinate of the point that corresponds to $v$ in $\Gamma$. We define

$$
\begin{aligned}
\operatorname{row}_{r_{i}} & =\left\{(x, y) \in R^{2} \mid x>x\left(r_{i+1}\right), y\left(r_{i}\right)<y<y\left(r_{i+1}\right)\right\}, \text { and } \\
\operatorname{col}_{r_{j}} & =\left\{(x, y) \in R^{2} \mid y>y\left(r_{j-1}\right), x\left(r_{j-1}\right)<x<x\left(r_{j}\right)\right\} .
\end{aligned}
$$

See also the left picture of Figure 2.
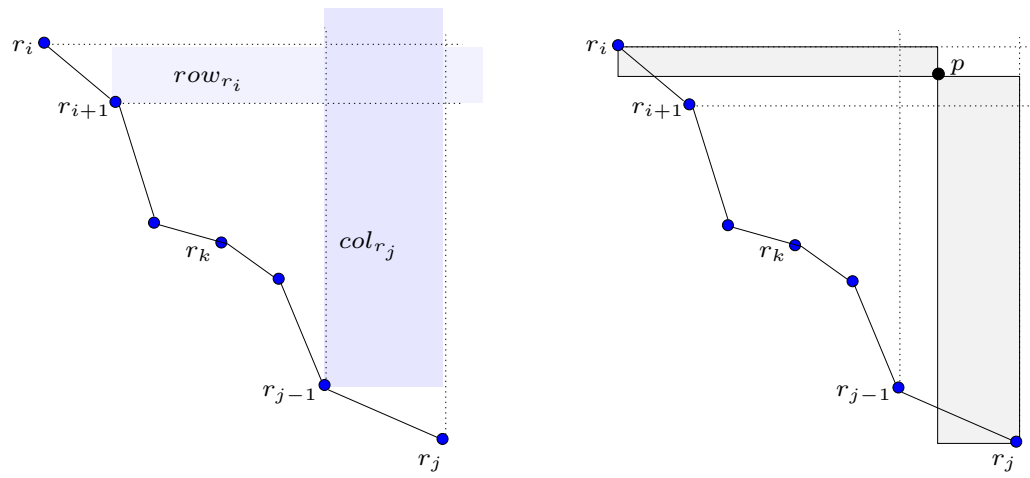

Fig. 2. Definition of $\operatorname{row}_{r_{i}}$ and $\operatorname{col}_{r_{j}}$. Placing a point $p$ in their intersection means that $p$ sees all vertices $r_{i}, r_{i+1}, \ldots, r_{j}$.

Lemma 2. If $p$ is a point in row $_{r_{i}} \cap \operatorname{col}_{r_{j}}$ for $i<j-1$, then $R\left[p, r_{k}\right]$ is empty for $i \leq k \leq j$.

Proof. For $i<k<j$, all points in $R\left[p, r_{k}\right]$ are above the ridge, therefore $R\left[p, r_{k}\right]$ is empty by the invariant. For $k=i$, note that $R\left[r_{i}, r_{i+1}\right]$ is empty because $\left(r_{i}, r_{i+1}\right)$ is an edge. Since all points in $R\left[p, r_{i}\right]$ are either above the ridge or in $R\left[r_{i}, r_{i+1}\right]$, it follows that $R\left[p, r_{i}\right]$ is empty. Similarly one shows that $R\left[p, r_{j}\right]$ is empty.

To explain the methods of adding vertices, we distinguish types of neighbours of the ridge-vertices.

Definition 4. Let the ridge degree, or $\operatorname{rdeg}(v)$, of a vertex $v \in V-V_{s}$ be the number of ridge vertices adjacent to $v$.

Definition 5. For $1 \leq i \leq l$, let $B_{i}$ be the neighbours of $r_{i}$ in $V-V_{s}$ that have ridge degree $\geq 2$ and are adjacent to a consecutive set of ridge-vertices starting at $r_{i}$. More formally, $B_{i}=\left\{v \in V-V_{s}\right.$ : there exists a $j>i$ such that the neighbours of $v$ in $R$ are $\left.r_{i}, r_{i+1}, \ldots, r_{j-1}, r_{j}\right\}$. Set $B=\bigcup_{i=1}^{l} B_{i}$. We call $a$ vertex $v$ a vertex of type $B$ if $v \in B$. 
Lemma 3. For $1 \leq i \leq l$, we have $\left|B_{i}\right| \leq 1$.

Proof. Assume that we had two vertices $b_{1}, b_{2}$ which both belong to $B_{i}$. By the definition of $G_{s}$, this means that they are both in the outer-face of $G_{s}$. And by Definition 5 both are adjacent to $r_{i}$ and $r_{i+1}$. One of these two 3-cycles must be filled, contradicting the assumption on the graph.

In the remainder of this section, we will denote by $b_{i}$ the (unique) vertex in $B_{i}$, if it exists.

Definition 6. For $1 \leq i \leq l$, let $C_{i}$ be the vertices in $V-V_{s}$ that are adjacent to $r_{i}$ and have ridge degree 1 . Set $C=\bigcup_{i=1}^{l} C_{i}$. We call a vertex $v$ a vertex of type $C$ if $v \in C$.

Definition 7. For $1 \leq i \leq l$, let $D_{i}$ be all those vertices in $V-V_{s}$ that are adjacent to $r_{i}$, but neither of type $B$ nor of type $C$. Set $D=\bigcup_{i=1}^{l} D_{i}$. We call a vertex $v$ a vertex of type $D$ if $v \in D$.

A vertex of type $D$ must have ridge degree $\geq 2$ (otherwise it would be of type $C$ ), and its neighbours on the ridge are not an interval on the ridge (otherwise it would be type $B$ ). In particular therefore, if $v \in D$, then there exists $i, j$ such that $j>i+1$ and $v$ is adjacent to $r_{i}$ and $r_{j}$, but not to $r_{i+1}$.

Placement I Assume there exists a vertex $v \in B$ such that $r \operatorname{deg}(v) \geq 3$.

Say, $v=b_{i}$, then by definition $b_{i}$ is adjacent to $r_{i}, r_{i+1}, \ldots, r_{j}$ for some $j>i+1$. Place $b_{i}$ in the intersection of $\operatorname{row}_{r_{i}}$ and $\operatorname{col}_{r_{j}}$. The new ridge is $R=$ $\left\{r_{1}, \ldots, r_{i}, b_{i}, r_{j}, \ldots, r_{l}\right\}$. By Lemma $2, b_{i}$ can see all of $r_{i}, \ldots, r_{j}$, so the new drawing is a planar cRI-drawing. One easily verifies the other conditions of the invariant.
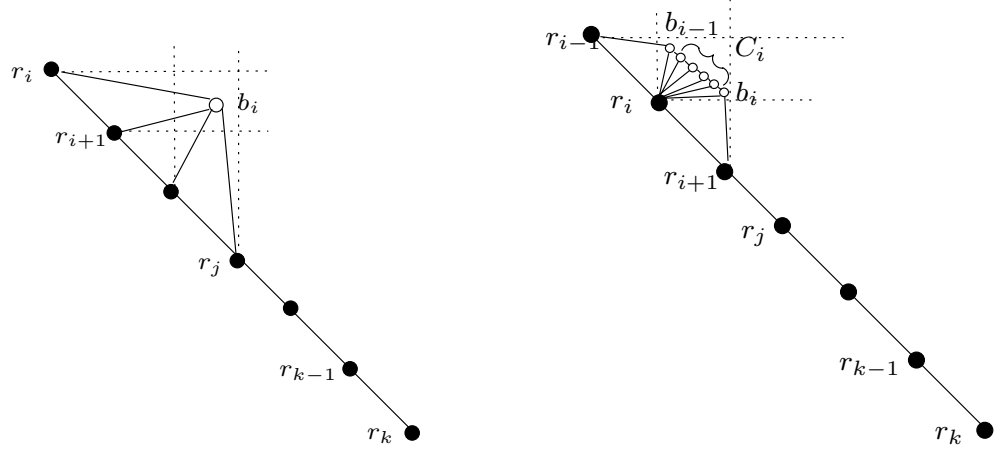

Fig. 3. Illustration of Placement I and Placement II.

Placement II Assume there exists an $1 \leq i \leq l$ such that $r_{i}$ is internal and $D_{i}=\emptyset$. 
Since $r_{i}$ is internal, the two edges $e_{0}=\left(r_{i-1}, r_{i}\right)$ and $e_{1}=\left(r_{i}, r_{i+1}\right)$ on the outer-face of $G_{s}$ are active and hence on the ridge, in particular therefore $i \geq 2$. Since $e_{0}$ and $e_{1}$ are active and $G$ is internally triangulated, there are vertices $x_{i-1}$ and $x_{i}$ in $V-V_{s}$ such that $x_{i-1}$ is adjacent to $r_{i-1}$ and $r_{i}$ and $x_{i}$ is adjacent to $r_{i}$ and $r_{i+1}$.

By definition, $\operatorname{rdeg}\left(x_{i-1}\right) \geq 2$ and $\operatorname{rdeg}\left(x_{i}\right) \geq 2$. Assume first $\operatorname{rdeg}\left(x_{i}\right) \geq 3$. Because $D_{i}=\emptyset, x_{i}$ must be of type $B$ or $C$, but by $\operatorname{rdeg}\left(x_{i}\right) \geq 3$ it must be of type $B$. So we have found a vertex of type $B$ with $\operatorname{rdeg}\left(x_{i}\right) \geq 3$, which means that we can apply Placement I. Similarly, we apply Placement I if $\operatorname{rdeg}\left(x_{i-1}\right) \geq 3$.

So we can place a vertex unless $\operatorname{rdeg}\left(x_{i-1}\right)=\operatorname{rdeg}\left(x_{i}\right)=2$. This implies that $x_{i-1}$ is incident only to $r_{i-1}$ and $r_{i}$ on the ridge, and $x_{i}$ is incident only to $r_{i}$ and $r_{i+1}$ on the ridge. Therefore $x_{i-1}=b_{i-1}$ and $x_{i}=b_{i}$, and in particular $x_{i-1} \neq x_{i}$.

Write $C_{i}=\left\{c_{1}, c_{2}, \ldots, c_{j}\right\}$ such that $\left\{b_{i-1}, c_{1}, \ldots, c_{j}, b_{i}\right\}$, are the neighbours of $r_{i}$ in $G-G_{s}$ in clockwise order; this is possible because $D_{i}=\emptyset$. Note that $C_{i}$ may be empty, in this case we have $j=0$. Let $b_{i-1}=c_{0}$ and $b_{i}=c_{j+1}$. Because $G$ is internally triangulated and $r_{i}$ is internal, the incident faces of $r_{i}$ are 3 -cycles, and therefore the edges $\left(c_{k}, c_{k+1}\right), 0 \leq k \leq j$ must exist. Place $\left\{b_{i-1}, c_{1}, \ldots, c_{j}, b_{i}\right\}$ on a diagonal within the intersection of row $_{r_{i-1}}$ and col $_{r_{i+1}}$ such that they are monotonically decreasing; see the right picture of Figure 3.

Since row $_{r_{i-1}} \cap \operatorname{col}_{r_{i+1}}$ was empty in $\Gamma$ and we placed the new vertices on a monotonically decreasing diagonal, $c_{k}$ can see $c_{k+1}$ for $0 \leq k \leq j$. Also, any $c_{k}$, $0 \leq k \leq j$ can see $r_{i}$; this holds because the configuration is simply Lemma 2 rotated by $\pi$. Notice that $\left\{b_{i-1}, c_{1}, \ldots, c_{j}, b_{i}\right\}$ has no chord, because any chord would induce a filled 3 -cycle between the two endpoints and $r_{i}$. Therefore, the new drawing is a planar cRI-drawing of the subgraph induced by the vertices. The new ridge is $R=\left\{r_{1}, \ldots, r_{i-1}, b_{i-1}, c_{1}, \ldots, c_{j}, b_{i}, r_{i+1}, \ldots, r_{l}\right\}$. One can verify the conditions of the invariant.

Proof of Correctness We now show that Placement I or Placement II is always possible. In fact, we show that Placement II (which possibly causes a Placement I) is always possible.

Lemma 4. Let $G$ be a biconnected, chordless, internally triangulated NF3-graph. Let $G_{s}$ be a strict subgraph of $G$, and let $\Gamma$ be a planar $c R I$-drawing of $G_{s}$ that satisfies the invariant with ridge $R=\left\{r_{1}, \ldots, r_{l}\right\}$. Then there exists a vertex $r_{i}$ that is internal and $D_{i}=\emptyset$.

Proof. We have two cases.

Case 1: $D=\emptyset$. Since $G_{s}$ is a strict subgraph of $G$, there exists a vertex $v$ on the outer-face of $G_{s}$ that has neighbours in $G-G_{s}$. At least one incident edge of $v$ on the outer-face of $G_{s}$ must be active, for if they were both inactive, then $v$ would be a cut-vertex of $G$, but $G$ is biconnected by assumption.

So assume $e=(v, w)$ is active. If neither $v$ nor $w$ are internal, then $e$ would be a chord of $G$, which is impossible because $G$ is chordless. So assume that one vertex, say $w$, is internal and by the invariant it belongs to the ridge, say $w=r_{i}$. Since $D$ is empty, so is $D_{i}$, and the claim follows. 
Case 2: $D \neq \emptyset$. Recall that for any $v \in D$ there exists $i, j$ such that $j>i+1$ and $v$ is adjacent to $r_{i}$ and $r_{j}$, but not to $r_{i+1}$. Among all possible $v \in D$ and all possible choices of such $i, j$ for $v$, choose a $v, i, j$ that minimize $j-i$.

We claim that $r_{i+1}$ is the desired vertex. Note that $r_{i+1}$ is internal: since $v$ is adjacent to $r_{i}$ and $r_{j}$ and there is at least one vertex in $V-V_{s}$ adjacent to $r_{i+2}$ we conclude that there is a path from $r_{i}$ to $r_{i+2}$ using only vertices in $V_{s}$. Also, $D_{i+1}$ must be empty. For assume there exists a $w \in D_{i+1}$, then $w \neq v$ because $w$ is adjacent to $r_{i+1}$ while $v$ is not. Therefore, all neighbours of $w$ must be inside the cycle $\left\{v, r_{i}, r_{i+1}, \ldots, r_{j}, v\right\}$. Let $j^{\prime}>i^{\prime}+1$ be such that $w$ is incident to $r_{i^{\prime}}$ and $r_{j^{\prime}}$, but not to $r_{i^{\prime}+1}$, then $i^{\prime} \geq i$ and $j^{\prime} \leq j$ by the above. In fact, $i^{\prime} \geq i+1$, because $w$ is incident to $r_{i+1}$. Therefore $j^{\prime}-i^{\prime}<j-i$, contradicting the choice of $i$ and $j$.

So $r_{i+1}$ is internal and $D_{i+1}=\emptyset$, and we have found the desired vertex.

Thus, as long as $G$ is not completed, we can always apply either Placement I or Placement II and obtain a planar cRI-drawing of a larger subgraph. This proves the following theorem:

Theorem 1. Any biconnected, chordless, internally triangulated NF3-graph G has a planar closed rectangle of influence drawing.

\subsection{Extension to all NF3-Graphs}

Lemma 5. If $G$ is an NF3-graph, then we can add edges to $G$ until we get a graph that is a biconnected, chordless, internally triangulated NF3-graph.

Proof. (sketch) We show first that if $G$ has a cutvertex or a chord, then we can always add an edge without creating a filled 3-cycle. Hence we can add edges to $G$ until it is biconnected and chordless and remains an NF3-graph. We can then, similarly to the method shown in [2], add edges to $G$ such that it remains a chordless NF3-graph and becomes internally triangulated.

Theorem 2. A graph admits a weak planar closed rectangle of influence drawing if and only if it is an NF3-graph.

Proof. The "only if" direction was shown in Lemma 1. The "if" direction follows from Theorem 1 and Lemma 5.

Corollary 1. Any NF3-graph $G$ has a planar open rectangle of influence drawing.

Proof. The proof follows from Theorem 2 and the fact that a closed rectangle of influence drawing is a weak open rectangle of influence drawing [8].

Corollary 2. Any 4-connected planar graph that is not triangulated has a planar closed rectangle of influence drawing.

Proof. This follows by choosing the outer-face of the graph to be the face that is not a 3-cycle; the drawing then has no filled 3-cycles and the graph is an NF3-graph. 


\section{Conclusion}

In this paper, we have studied rectangle of influence drawings, and have shown that a planar graph has a weak planar closed rectangle of influence drawing if and only if it can be embedded without filled 3-cycles. In particular, all 4-connected planar graphs that are not triangulated have such a drawing.

We conclude with remarks and pointers to open problems:

- Some planar graphs (for example $K_{4}$ ) have an open planar rectangle of influence graphs even though they are not an NF3-graph. But other planar graphs (for example the octahedron) can be shown not to have such a drawing. Can we characterize those planar graphs that have a planar open rectangle of influence drawing? Can we find an algorithm to determine whether a planar graph has such a drawing?

- Does every planar graph have a weak open rectangle of influence drawing (it need not be planar)? How about closed rectangle of influence drawings?

- Does every NF3-graph have a strong rectangle of influence drawing?

- In our construction, no two vertices are ever placed in the same row or in the same column. By applying an order-preserving transformation, we thus obtain a drawing in an $n \times n$-grid. But one vertex per row/column seems a waste. Can we show that every NF3-graph has a rectangle of influence drawing of area significantly less than $n^{2}$ ?

\section{References}

1. N. Alon, Z. Füredi, and M. Katchalski. Separating pairs of points by standard boxes. European Journal of Combinatorics, 6:205-210, 1985.

2. T. Biedl, G. Kant, and M. Kaufmann. On triangulating planar graphs under the four-connectivity constraint. Algorithmica, 19(4):427-446, 1997.

3. J. Bondy and U. Murty. Graph Theory and Applications. Amerian Elsevier Publishing Co., 1976.

4. M. de Berlin, S. Carlsson, and M. Overmars. A general approach to dominance in the plane. Journal of Algorithms, 13:274-296, 1992.

5. G. Di Battista, W. Lenhart, and G. Liotta. Proximinity drawability: A survey. In R. Tamassia and I. Tollis, editors, DIMACS International Workshop, Graph Drawing 94, volume 894 of Lecture Notes in Computer Science, pages 328-339. Springer-Verlag, 1995.

6. H. de Fraysseix, J. Pach, and R. Pollack. How to draw a planar graph on a grid. Combinatorica, 10:41-51, 1990.

7. M. Ichino and J. Sklansky. The relative neighborhood graph for mixed feature variables. Pattern Recognition, 18(2):161-167, 1985.

8. G. Liotta, A. Lubiw, H. Meijer, and S. Whitesides. The Rectangle of Influence drawability problem. Computational Geometry: Theory and Applications, 10(1):122, 1998.

9. M.H. Overmars and D. Wood. On rectangular visibility. Journal of Algorithms, 9(3):372-390, 1988.

10. F. Preparata and M. Shamos. Computational Geometry: An Introduction. SpringerVerlag, New York, 1985. 